\title{
Steroid-triggered, cell-autonomous death of a Drosophila motoneuron during metamorphosis
}

\author{
Ari Winbush ${ }^{1,2}$ and Janis C Weeks ${ }^{1 *}$
}

\begin{abstract}
Background: The metamorphosis of Drosophila melanogaster is accompanied by elimination of obsolete neurons via programmed cell death (PCD). Metamorphosis is regulated by ecdysteroids, including 20-hydroxyecdysone (20E), but the roles and modes of action of hormones in regulating neuronal PCD are incompletely understood.

Results: We used targeted expression of GFP to track the fate of a larval motoneuron, RP2, in ventral ganglia. RP2s in abdominal neuromeres two through seven (A2 to A7) exhibited fragmented DNA by 15 hours after puparium formation (h-APF) and were missing by 20 h-APF. RP2 death began shortly after the 'prepupal pulse' of ecdysteroids, during which time RP2s expressed ecdysteroid receptors (EcRs). Genetic manipulations showed that RP2 death required the function of EcR-B isoforms, the death-activating gene, reaper (but not hid), and the apoptosome component, Dark. PCD was blocked by expression of the caspase inhibitor p35 but unaffected by manipulating Diap1. In contrast, aCC motoneurons in neuromeres A2 to A7, and RP2s in neuromere A1, expressed EcRs during the prepupal pulse but survived into the pupal stage under all conditions tested. To test the hypothesis that ecdysteroids trigger RP2's death directly, we placed abdominal GFP-expressing neurons in cell culture immediately prior to the prepupal pulse, with or without 20E. 20E induced significant PCD in putative RP2s, but not in control neurons, as assessed by morphological criteria and propidium iodide staining.

Conclusions: These findings suggest that the rise of ecdysteroids during the prepupal pulse acts directly, via EcR-B isoforms, to activate PCD in RP2 motoneurons in abdominal neuromeres A2 to A7, while sparing RP2s in A1. Genetic manipulations suggest that RP2's death requires Reaper function, apoptosome assembly and Diap1independent caspase activation. RP2s offer a valuable 'single cell' approach to the molecular understanding of neuronal death during insect metamorphosis and, potentially, of neurodegeneration in other contexts.
\end{abstract}

\section{Background}

Metamorphosis of the fruit fly, Drosophila melanogaster, entails the transformation of a crawling, feeding larva into an adult capable of flight and reproduction. During this process the larval nervous system is reorganized to accommodate new adult-specific behaviors through neuronal remodeling, the development of previously quiescent adult-specific imaginal neurons, and the elimination of obsolete larval neurons by programmed cell death (PCD) [1,2]. Neuronal death likewise accompanies embryogenesis, but the extent to which underlying causes and mechanisms of neuronal PCD are shared

\footnotetext{
*Correspondence: jweeks@uoregon.edu

'Department of Biology, Institute of Neuroscience, University of Oregon Eugene, OR, 97403-1254, USA

Full list of author information is available at the end of the article
}

during these two periods, or with PCD in other cell types, is largely unexplored $[3,4]$.

Metamorphosis is regulated in large part by steroid hormones termed ecdysteroids, including the active metabolite, 20-hydroxyecdysone (20E). 20E exerts its actions via three isoforms of the ecdysteroid receptor (EcR): EcR-A, EcR-B1 and EcR-B2 [5]. The specific sequence of hormonal changes that drive metamorphosis in Drosophila are shown in Figure 1. A 'late larval pulse' transforms the wandering larva into a white prepupa at puparium formation. A smaller 'prepupal pulse' approximately 10 hours after puparium formation (hAPF) initiates pupation, whose hallmark, head eversion, occurs at approximately $12 \mathrm{~h}$-APF. A prolonged 'pupal pulse' beginning at approximately $24 \mathrm{~h}$-APF drives the remaining development of the adult fly. As adult

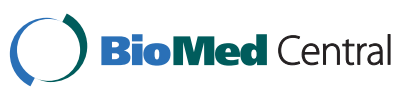




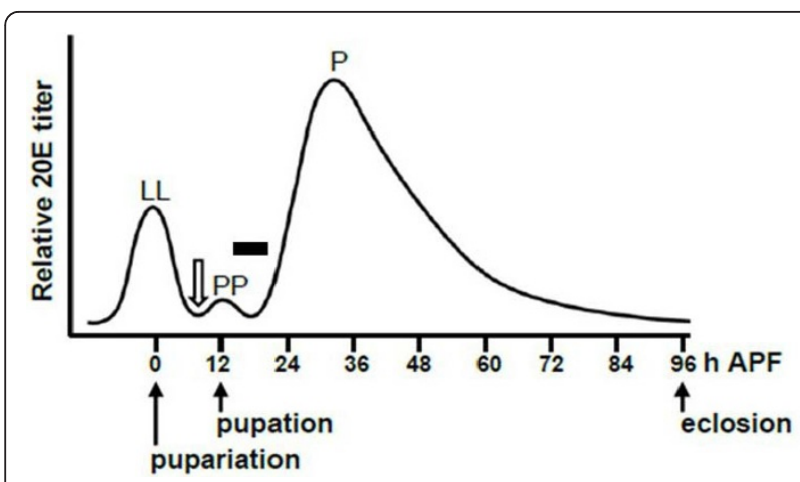

Figure 1 Endocrine events during Drosophila metamorphosis Timeline illustrates changes in ecdysteroid levels during the larvalpupal-adult transformation at $25^{\circ} \mathrm{C}$. The late larval (LL) pulse of 20hydroxyecdysone (20E) triggers pupariation, with subsequent development timed by hours after puparium formation (h-APF). The prepupal (PP) pulse at approximately $12 \mathrm{~h}$-APF triggers pupation; the pupal $(P)$ pulse drives development of the adult fly. Emergence of the adult (eclosion) occurs at approximately 96 h-APF. Open arrow indicates when neuronal cultures were prepared (8 h-APF; see Results). Filled bar shows period when abdominal RP2s undergo PCD (approximately 14 to 20 h-APF; see Results). Adapted from [12] based on hormone titers from $[7,72]$.

eclosion (emergence) approaches, ecdysteroids decline and remain low or absent during early adult life [6,7].

A role for ecdysteroids in postembryonic neuronal remodeling and PCD has been demonstrated in Drosophila and in another well-studied insect species, the hawkmoth Manduca sexta [1]. In Drosophila, the remodeling of larval motoneurons, mushroom body neurons and FMRFamide-expressing thoracic ventral neurons is regulated by the late larval pulse and pupal pulse of ecdysteroids [8-16]. The PCD of a subset of larval ventral neurons that express the neuropeptide Corazonin (vCrz) appears to be triggered by the late larval pulse, via EcR-B isoforms [17]. In Manduca, the remodeling or death of larval abdominal motoneurons is regulated by the prepupal and pupal pulses of ecdysteroids [18-23]. The decline in ecdysteroids at the end of the pupal stage in both Drosophila and Manduca triggers PCD of neurons not needed during the adult stage[2,24,25]. Drosophila neurons in this category include 'type II' neurons identifiable by their strong EcR-A expression, and a subset of neurons that express the neuropeptide crustacean cardioactive peptide [26,27]. The death of type II and crustacean cardioactive peptide neurons requires both an ecdysteroid decline and an unidentified descending signal [25-28]. Manduca likewise has neurons that require these two cues to initiate PCD after eclosion $[29,30]$.

In Drosophila, PCD has been shown to be controlled through a balance between death-activating and deathinhibiting proteins. The core cell death machinery includes effector caspases which, following their activation by initiator caspases, degrade cellular proteins [31]. In Drosophila, the initiator caspase Dronc (Drosophila Nedd2-like caspase) likely governs most PCD [32-36]. Dronc autoprocessing and activation requires recruitment into an apoptosome formed by the Drosophila Apaf-1related killer (Dark) protein [37-40]. Animals carrying dark mutations show PCD defects similar to those seen in dronc mutants [41-43]. Caspases are normally inhibited by the Drosophila inhibitor of apoptosis protein (Diap1) through ubiquitination [44-48]. The canonical cell death pathway involves the inactivation and degradation of Diap1 by proteins encoded by the genes reaper, hid, and grim [49-51]. The inactivation of Diap1 by Reaper, Hid or Grim allows Dronc to accumulate and be recruited into apoptosomes [38,44,52,53].

In Manduca, the most detailed information regarding ecdysteroid-induced neuronal PCD has come from the segment-specific death of larval accessory planta retractor (APR) motoneurons during metamorphosis [20]. The death of a segmental subset of APRs at pupation is triggered directly and cell-autonomously by the rise in ecdysteroids during the prepupal peak, as demonstrated by placing the motoneurons in cell culture and exposing them to appropriate changes in 20E levels [54-56]. Likewise, PCD of the remaining APRs, which occurs following eclosion, is a direct, cell-autonomous response to the decline of ecdysteroids at the end of the pupal stage [24].

Delineation of molecular pathways by which ecdysteroids activate postembryonic PCD ideally requires an experimental system in which neurons of interest are direct targets of ecdysteroids, genes/proteins involved in PCD are known, and the EcRs and death genes can be genetically manipulated. The APRs of Manduca fulfill the first criterion but largely lack the other features. In contrast, Drosophila offers the latter two features but lacks identified neurons whose deaths unambiguously result from direct ecdysteroid action. The present experiments made use of RP2 motoneurons, which have been studied extensively in other contexts [57-59]. We found that a segmental subset of RP2s, located in abdominal neuromeres $\mathrm{A} 2$ to $\mathrm{A} 7$, undergoes $\mathrm{PCD}$ at the onset of metamorphosis in direct response to the rise in ecdysteroids during the prepupal pulse, and that EcRs and previously identified death genes participate in RP2's demise. This experimental system provides an opportunity to investigate the chain of events linking steroid hormone action to the PCD of an individually identified neuron during postembryonic development.

\section{Results}

GFP labeling to track neurons during metamorphosis

We used the Gal4/UAS system combined with FLP/ FRT-mediated excision (see Materials and methods) to maintain postembryonic expression of membrane-bound 
GFP in RP2 and aCC (anterior corner cell) motoneurons and, less strongly (see below), pCC (posterior corner cell) interneurons. These neurons occur in segmentally repeated, bilateral pairs along the midline of the ventral ganglion and are readily identifiable in situ by their characteristic locations and projections [59-61] (Figure 2A). Their somata lie near the dorsal surface of the ventral ganglion, with aCCs and pCCs located immediately anterior to the RP2s of the next posterior neuromere. The motoneurons' axons exit the central nervous system via the ipsilateral intersegmental nerve (Figure $2 \mathrm{~A}$ ). In third instar larvae and young prepupae, GFP-labeled RP2s, aCCs and pCCs were present in both thoracic and abdominal neuromeres (data not shown) but, for simplicity, we restricted the present study to abdominal neuromeres A1 to A7.

The reporter lines used for this study produced two distinct patterns of neuronal labeling in third instar larvae and young prepupae, prior to the onset of metamorphic changes; conveniently, one pattern was advantageous for in vivo experiments while the other

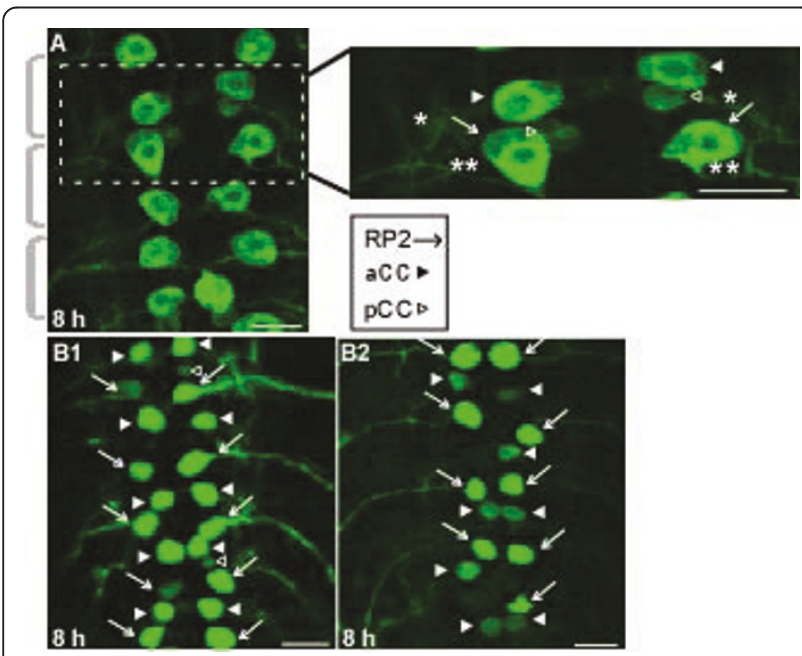

Figure 2 GFP expression to identify and track neurons. (A) Left: confocal image showing a repeating pattern of GFP-labeled neurons along the anterior-posterior axis of the abdominal region of a ventral ganglion at 8 h-APF. Grey brackets denote neuromeres. For this and all subsequent confocal images, abdominal neuromeres are shown with anterior up, h denotes h-APF, and RP2s, aCCs and pCCs are marked by arrows, filled arrowheads, and open arrowheads, respectively (see key below inset). The dashed box outlines the enlarged inset at right, illustrating GFP labeling in bilateral pairs of aCCs, RP2s and more faintly labeled pCCs. aCC axons (single asterisks) project posteriorly to join ipsilateral intersegmental nerves (ISNs) while RP2 axons (double asterisks) project anteriorly to join the ISNs. pCC axons are not indicated. (B) Comparison of RP2/aCC-enhanced (B1) and RP2-enhanced (B2) GFP labeling patterns (see Results) at 8 h-APF. The RP2/aCC-enhanced pattern was used to track neurons through metamorphosis; the RP2-enhanced pattern was used for cell culture experiments. Scale bars: $15 \mu \mathrm{m}$. was advantageous for cell culture experiments. All animals were reared at $25^{\circ} \mathrm{C}$ unless indicated otherwise. In the first pattern ('RP2/aCC-enhanced'), GFP labeling was strong in both RP2s and aCCs, with all or nearly all of these motoneurons labeled throughout the abdominal central nervous system (for example, Figures 2A, B1 and $3 \mathrm{~A}$; Table 1). There was also variable and weaker labeling of a small proportion of pCC interneurons and some unidentified neurons (for example, Figures 2A, B1 and $3 \mathrm{~A} 3$ ). The RP2/aCC-enhanced labeling pattern was observed in three conditions: all animals with two copies of $U A S-F L P, A c t 5 C>y+>$ Gal4 on chromosome 2 or 3; all animals with one copy of UAS-FLP, Act $5 C>y+>$ Gal4 on chromosome 2 or 3 , reared at $29^{\circ} \mathrm{C}$ until the third instar; or in approximately half [62] of animals in which UAS-FLP was on chromosome 1 (see below). Animals with the RP2/aCC-enhanced phenotype from the first two categories were used to track neuronal fates during metamorphosis.

In the second labeling pattern ('RP2-enhanced'), RP2s were strongly GFP-labeled in third instar larvae and young prepupae, but the frequency and intensity of labeling of aCCs, pCCs and unidentified neurons were reduced compared to the $\mathrm{RP} 2 / \mathrm{aCC}$-enhanced pattern. The RP2-enhanced labeling pattern occurred in approximately half the animals with $U A S-F L P$ on chromosome 1 (see above) [62]. Figure 2B compares the two GFP labeling patterns observed in animals of this genotype; we did not investigate potential causes of the two labeling patterns. Animals with the RP2-enhanced GFP labeling pattern were selectively used for cell culture experiments investigating RP2's response to 20E (see below).

In both labeling patterns, GFP labeling was sometimes absent from individual neurons, including RP2s (for example, Figures 2B2 and 3A; Table 1), perhaps due to variable efficacy of the Gal4/UAS and FLP/FRT method $[58,63]$.

\section{Loss of RP2s during metamorphosis occurs by programmed cell death}

Previous reports documented an intense period of PCD in the ventral ganglion during the first $24 \mathrm{~h}-\mathrm{APF}[2,17]$. To investigate RP2 and aCC fates during this period, we first compared ventral ganglia at 8 and $30 \mathrm{~h}$-APF; in this and all subsequent in vivo experiments, only animals with the RP2/aCC-enhanced GFP labeling pattern were used. At $8 \mathrm{~h}$-APF, the repeating segmental pattern of RP2s and aCCs was observed in 100\% of ventral ganglia ( $n=23$ animals; for example, Figures 2 and $3 \mathrm{~B}$ ); in contrast, in all ventral ganglia examined at $30 \mathrm{~h}-\mathrm{APF}$, aCCs were present while RP2s were missing, except in neuromere A1 ( $\mathrm{n}=18$ animals) [62]. To investigate this phenomenon more precisely, we quantified the number 


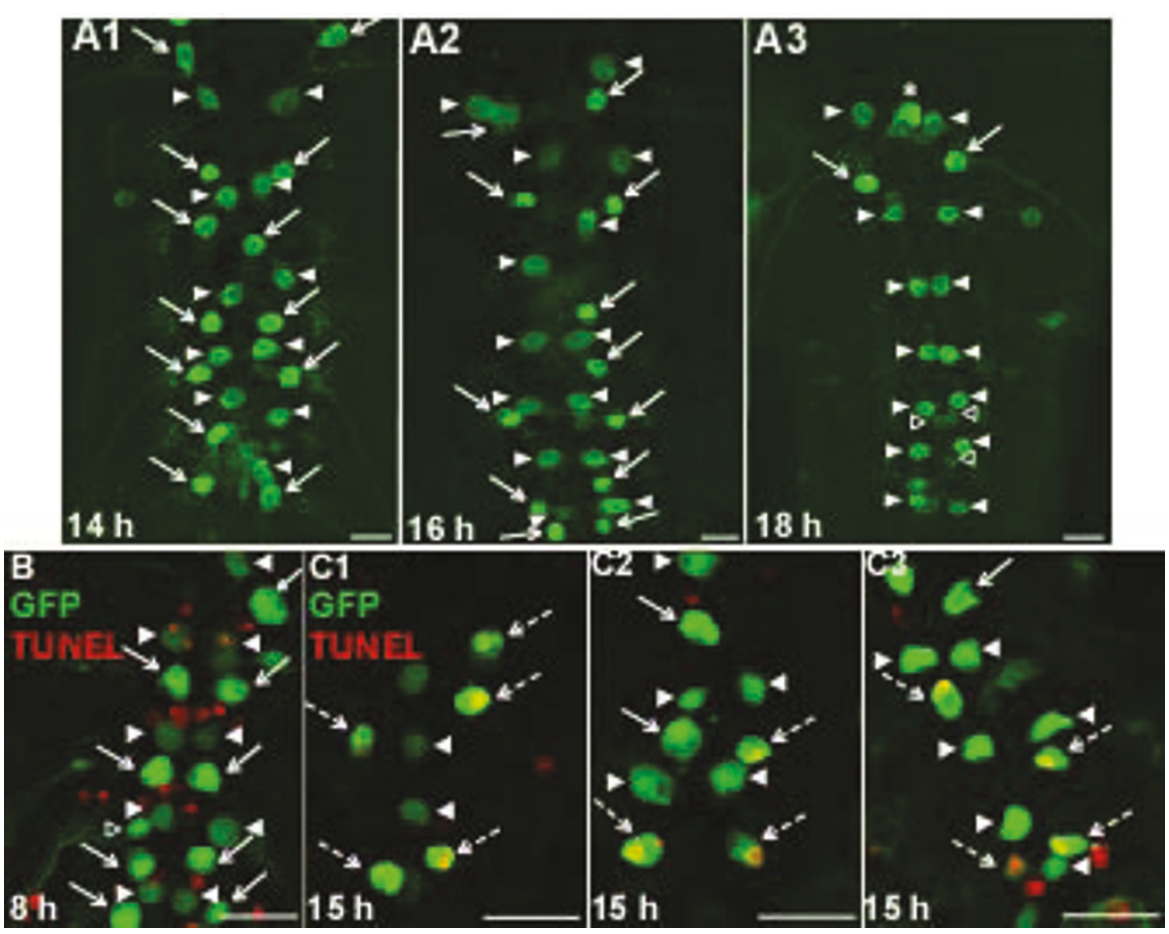

Figure 3 RP2 disappearance occurs by programmed cell death. (A) Images showing ventral ganglia removed from animals at 14, 16 and 18 h-APF. At $14 \mathrm{~h}$-APF, nearly the full complement (2 per neuromere; Table 1) of abdominal RP2s and aCCs were identifiable by location and GFP labeling. By 16 h-APF, many RP2s were missing or were rounded and condensed. By 18 h-APF, only the RP2s in neuromere A1 remained. In contrast, aCCs persisted throughout this period. Asterisk in (A3) marks an example of an unidentified GFP-labeled neuron. (B, C) GFP labeling (green) and TUNEL labeling (indicating DNA fragmentation; red) in mid-abdominal neuromeres. (B) At 8 h-APF, many TUNEL-positive nuclei were present but no RP2s or aCCs were TUNEL-positive. (C1-C3) Images from three different animals at 15 h-APF. TUNEL-positive RP2s are marked by dashed arrows. Scale bars: $20 \mu \mathrm{m}$.

of RP2s and aCCs in neuromeres A1 to A7 at 2-hour intervals from 12 to $20 \mathrm{~h}$-APF (Table 1). In A1, the maximum possible number of GFP-labeled RP2s and aCCs was two each; however, because GFP labeling was occasionally absent (see above), the mean number of RP2s and aCCs in A1 was slightly less than two. These

Table 1 Number of GFP-labeled motoneurons (mean \pm standard deviation) in abdominal neuromeres $A 1$ and A2 to A7 from 12 to 20 h-APF.

\begin{tabular}{lcccc}
\hline & \multicolumn{2}{c}{ Neuromere A1 } & \multicolumn{2}{c}{ Neuromeres A2 to A7 } \\
\cline { 2 - 5 } & aCC & RP2 & aCC & RP2 \\
\hline 12 h-APF (14) & $1.9 \pm 0.3$ & $1.9 \pm 0.3$ & $11.6 \pm 0.6$ & $11.8 \pm 0.6$ \\
14 h-APF (20) & $2.0 \pm 0.0$ & $1.9 \pm 0.3$ & $10.9 \pm 1.1$ & $10.7 \pm 1.6$ \\
16 h-APF (21) & $1.9 \pm 0.2$ & $1.8 \pm 0.4$ & $10.4 \pm 1.0$ & $4.6 \pm 3.1^{\mathrm{a}}$ \\
18 h-APF (16) & $1.9 \pm 0.3$ & $1.9 \pm 0.3$ & $10.6 \pm 1.1$ & $2.2 \pm 2.5^{\mathrm{a}}$ \\
20 h-APF (10) & $2.0 \pm 0.0$ & $2.0 \pm 0.0$ & $10.6 \pm 1.0$ & $0.1 \pm 0.3^{\mathrm{a}}$ \\
\hline
\end{tabular}

The maximum possible total number of motoneurons was two aCCs and two RP2s in A1, and 12 aCCs and 12 RP2s in A2 through A7. Number of animals $(\mathrm{n})$ is given in parentheses. ${ }^{\mathrm{a}}$ Significant difference between number of aCC and RP2 motoneurons in A2 through A7, at the times shown $(P<0.0001$; Student's paired $t$-test, two-tailed); the number of RP2s was first significantly reduced at $16 \mathrm{~h}$-APF. The number of aCC and RP2 motoneurons in A1 was stable over this time period. values did not change between 12 and $20 \mathrm{~h}$-APF (Table 1), confirming that RP2s and aCCs persisted in A1 throughout this period. A different pattern was observed in neuromeres $\mathrm{A} 2$ to $\mathrm{A} 7$, in which the maximum possible total number of GFP-labeled RP2s and aCCs was 12 each. Between 12 to $20 \mathrm{~h}$-APF, the number of aCCs remained stable while the number of RP2s declined precipitously, with the largest loss occurring between 14 and $16 \mathrm{~h}$-APF (Table 1). Representative images of these patterns are shown in Figure 3A. During the peak period of RP2 loss, many RP2s appeared rounded and condensed (for example, Figure 3A2), consistent with the possibility that these neurons were dying.

To test the hypothesis that the loss of RP2s between 14 and $20 \mathrm{~h}$-APF resulted from PCD rather than, for example, loss of GFP expression, we used the TUNEL method (terminal deoxynucleotidyl transferase mediated dUTP nick end labeling; see Materials and methods) to label fragmented DNA, a hallmark of PCD [64]. Ventral ganglia examined between 6 and $10 \mathrm{~h}$-APF exhibited many TUNEL-positive nuclei, but no TUNEL-positive RP2s (for example, Figure 3B; $n=6$ animals). However, at $15 \mathrm{~h}$-APF, every ventral ganglion examined had one 
or more TUNEL-positive RP2s (Figure 3C; $\mathrm{n}=10$ animals). At no time during the observation period did we see TUNEL-positive aCCs ( $\mathrm{n}=16$ animals). We conclude from these data that RP2s in neuromeres A2 to A7 underwent PCD between approximately 14 and 20 h-APF. In contrast, the RP2s in A1, and all abdominal aCCs, survived, thereby serving as convenient positive controls in subsequent experiments.

\section{Genes and proteins involved in RP2 death}

To further characterize the death of RP2s during metamorphosis, we tested for the involvement of a subset of the many genes and proteins implicated in PCD in Drosophila [31]. The PCD of cells, including neurons $[26,65]$, depends on proteins encoded by the death-activating genes reaper, hid, and grim [49-51]. We therefore tested whether these genes were involved in the death of RP2s. In an H99/+ mutant background in which reaper, hid, and grim were reduced to one copy [51], RP2s in neuromeres A2 to A7 died normally; they were present at $8 \mathrm{~h}$-APF (Figure $4 \mathrm{~A} 1 ; \mathrm{n}=5$ animals) but absent by $24 \mathrm{~h}$-APF, while all aCCs survived (Figure 4A2; $\mathrm{n}=17$ animals). Likewise, in a hid ${ }^{\text {P05014 }} /$ H99 background that removes hid function and reduces reaper and grim to one copy[50,51,51], RP2s in A2 to A7 died while the aCCs survived normally (Figure 4B; $n=12$ animals). In contrast, in $D f(3 L) X R 38 / H 99$ animals, in which reaper was eliminated and hid and grim were reduced to one copy [65], RP2s failed to undergo PCD and were present along with aCCs at $24 \mathrm{~h}$-APF (Figure $4 C ; n=9$ animals). In the above experiments, RP2s in neuromere A1 behaved like aCCs (data not shown). These results suggested an exclusive requirement for reaper in the PCD of RP2s in neuromeres A2 to A7.

In most instances of developmental PCD, Reaper, Grim, and/or Hid normally antagonize Diap1 to liberate the apical caspase Dronc, which in turn activates

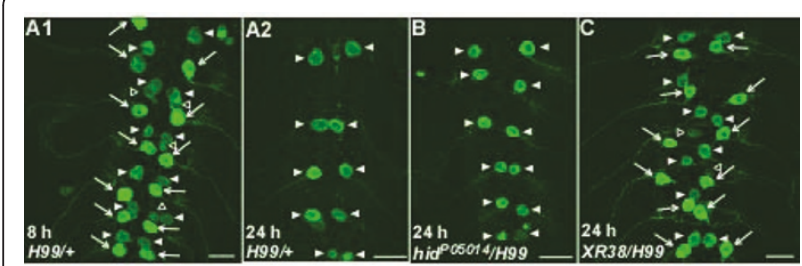

Figure 4 Reaper is necessary for RP2 death. (A) H99/+ animals (heterozygous for a deletion that removes reaper, hid, and grim) showed normal patterns of GFP-labeled neurons at 8 h-APF (A1) and RP2 death and aCC survival at 24 h-APF (A2). (B) A hid P05014/ H99 animal (hid loss of function mutation) showed normal RP2 death and aCC survival at 24 h-APF. (C) In a XR38/H99 animal (reaper deletion), RP2s failed to undergo PCD and were present along with aCCs at 24 h-APF. All images are from mid-abdominal neuromeres. Scale bars: $20 \mu \mathrm{m}$. effector caspases such as Drice [31,46,66,67]. Given the requirement of Reaper for RP2's death, we tested whether ectopic expression of Diap1 in RP2s would block PCD - for example, as it does in larval Drosophila salivary glands [67]. In UAS-Diap1 animals, all RP2s were present at $8 \mathrm{~h}-\mathrm{APF}$, as expected (Figure 5A1; $\mathrm{n}=5$ animals). At $20 \mathrm{~h}$-APF, RP2s in A2 to A7 were missing while aCCs survived (Figure 5A2; $\mathrm{n}=15$ animals), suggesting that Diap1 expression could not antagonize the death of RP2s. As a positive control, we replicated the finding that ectopic expression of Diap1 in larval salivary glands prevented degeneration (data not shown) [62]. We further investigated the involvement of Diap1 in animals homozygous for a dominant diap1 mutation, thread ${ }^{S L}\left(t h^{S L}\right)$. The $t h^{S L}$ allele produces a Diap1 protein with an amino acid substitution in the BIR-1 domain, which suppresses Diap1 inactivation by Reaper [68]. However, RP2 death proceeded normally in $t h^{S L}$ homozygotes (Figure 5A3; $\mathrm{n}=8$ animals), just as in UAS-Diap1 animals. In all cases, RP2s in neuromere A1 behaved like aCCs (data not shown). These results, using two independent genetic manipulations, suggested that Diap1 may not play a role in the death of RP2s in A2 to A7; a similar result was reported for the Reaperdependent PCD of vCrz neurons during Drosophila metamorphosis [17].

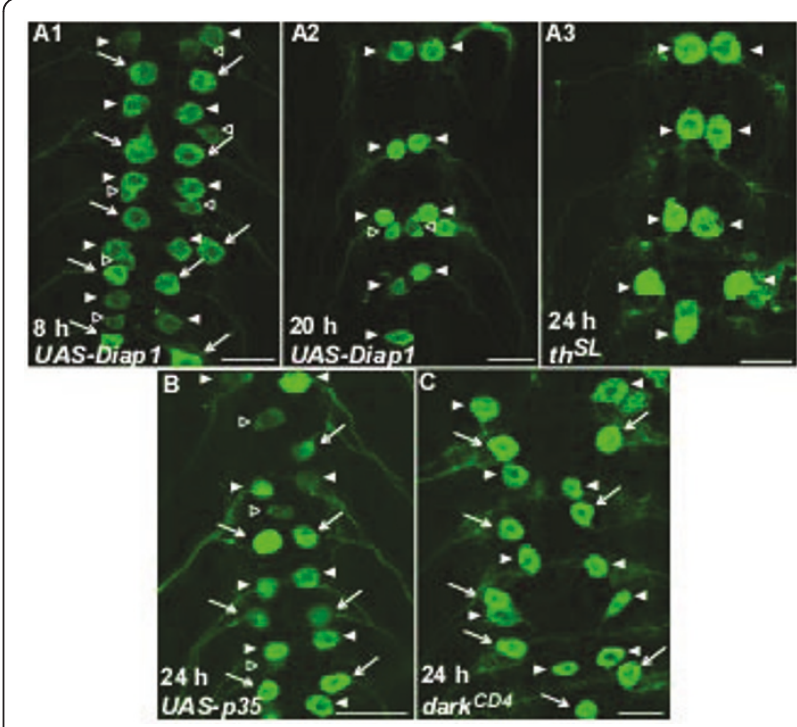

Figure 5 Effects of perturbing Diap1, p35 and Dark on RP2 death. (A) UAS-Diapl animals showed normal patterns of GFPlabeled neurons at 8 h-APF (A1) and RP2 death and aCC survival at 20 h-APF (A2). (A3) RP2 death was likewise unimpaired in $t h^{S L}$ mutants, in which Diap1 inactivation by Reaper is suppressed. (B) Expression of p35 via UAS-p35 blocked the death of RP2s, which were still present at 24 h-APF. (C) In a dark ${ }^{C D 4} /$ dark $^{C D 4}$ animal (homozygous for a dark hypomorphic allele), RP2s were still present at $24 \mathrm{~h}$-APF. All images are from mid-abdominal neuromeres. Scale bars: $20 \mu \mathrm{m}$. 
To test the roles of caspases in RP2 death, we mis-expressed the viral caspase inhibitor p35 using a $U A S-p 35$ responder. p35 blocks PCD by acting as a cleavage substrate for the effector caspase Drice $[69,70]$. In the UAS-p35 genetic background, RP2s were present at $8 \mathrm{~h}$-APF, as expected ( $\mathrm{n}=11$ animals; data not shown). At 24 h-APF, RP2s were still present in all UAS-p35 animals examined (Figure 5B; $\mathrm{n}=17$ animals), consistent with a requirement for effector caspase activity for the PCD of RP2s.

Activation of the apical caspase Dronc requires assembly of the Drosophila Apaf-1 homologue, Dark [71], into apoptosomes, with dark mutations phenocopying null mutations in dronc [41] We tested the requirement for dark in RP2 death using animals homozygous for the hypomorphic $\operatorname{dark}^{C D 4}$ allele. At $24 \mathrm{~h}-\mathrm{APF}, \mathrm{RP} 2 \mathrm{~s}$ were still present in dark $^{C D 4}$ mutants (Figure $5 \mathrm{C}$; $\mathrm{n}=8$ animals), suggesting that apoptosome assembly involving Dark and Dronc is required for the PCD of RP2s.

In summary, of the subset of Drosophila PCD-related genes and proteins that we tested, several were implicated in the death of RP2s in neuromeres A2 to A7 during metamorphosis. The only exception was Diap1 (see Discussion).

\section{RP2 death is triggered directly and cell-autonomously by $20 \mathrm{E}$}

The time course of the death of RP2s in neuromeres A2 to A7 (Table 1 ) closely matches that of the degeneration of larval salivary glands, which was shown in tissue culture to be triggered directly by the rise of ecdysteroids during the prepupal pulse [66]. In Manduca, the segment-specific PCD of APR motoneurons at pupation is likewise triggered directly and cell-autonomously by the rise in ecdysteroids during the prepupal pulse, as demonstrated by placing fluorescently labeled APRs in primary cell culture and exposing them to 20E [56]. We therefore tested the hypothesis that prepupal ecdysteroids act directly on Drosophila RP2s to trigger PCD.

If ecdysteroids act directly on RP2s to activate PCD, then RP2s should express ecdysteroid receptors at the appropriate time. Immunolabeling showed that both EcR-A and EcR-B1 were present in the nuclei of RP2s and aCCs in all abdominal segments at 8 to $10 \mathrm{~h}-\mathrm{APF}$, during the initial upswing of the prepupal pulse (Figure 6A, B; $n$ $=6$ and 5 animals, respectively). EcR labeling was qualitatively similar in all RP2s and aCCs, although only the RP2s in neuromeres A2 to A7 were fated to die. Levels of EcR-A and EcR-B1 in RP2s appeared low relative to their levels in co-processed ventral ganglia from late pupae or white prepupae (data not shown) [27,72]. Control experiments with primary antibody omitted eliminated labeling (data not shown). Antibodies specific to EcR-B2 are unavailable so this isoform was not examined.

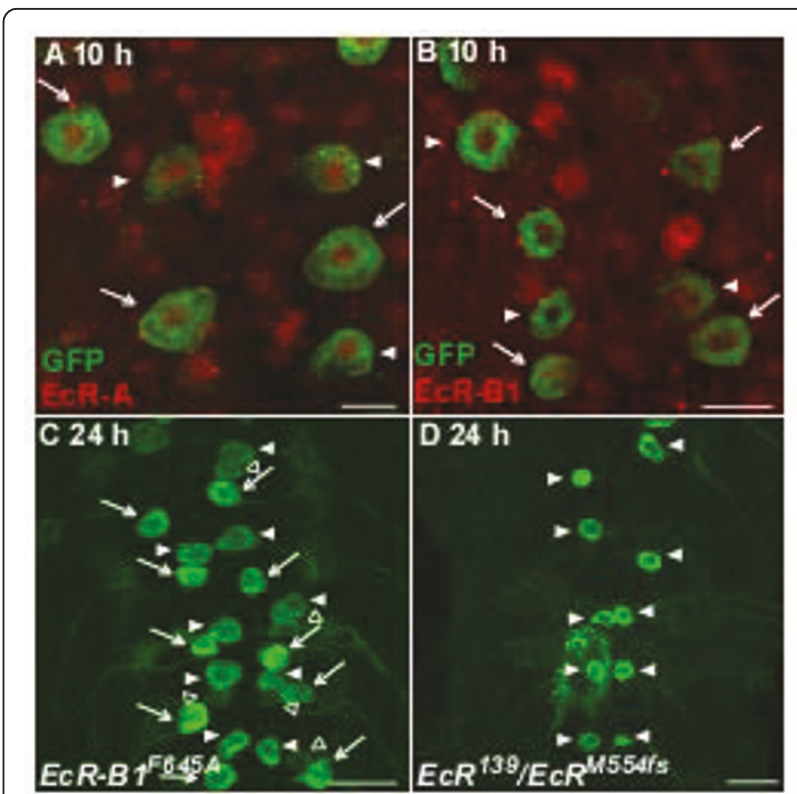

Figure 6 RP2 death requires ecdysteroid receptor function (A, B) GFP labeling (green) and EcR immunoreactivity (red). At 10 h-APF, just prior to head eversion, both RP2s and aCCs exhibited low levels of nuclear ECR-A (A) and EcR-B1 (B). (C) In UAS-ECR-B1 ${ }^{F 645 A}$ (dominant-negative ECR-B1 mutation expressed in the neurons), RP2s were still present at $24 \mathrm{~h}-\mathrm{APF}$. (D) $E C R^{139} / E C R^{M 5545 s}$ (ECR-A null mutation) animals showed normal RP2 death and aCC survival at 24 h-APF. Scale bars: $10 \mu \mathrm{m}$ in (A, B); $20 \mu \mathrm{m}$ in (C, D).

We next tested the requirement for EcRs in RP2's death by misexpressing a dominant-negative EcR-B1 mutation that binds ecdysteroids but lacks transcriptional activation $\left(U A S-E c R-B 1^{F 645 A}\right)[8,73]$. This mutation competitively displaces all three endogenous EcR isoforms so cannot distinguish the roles of individual isoforms. In $U A S-E c R-B 1^{F 645 A}$ animals, RP2s failed to undergo PCD and were present along with aCCs when examined at $24 \mathrm{~h}$-APF (Figure 6C; $\mathrm{n}=9$ animals), suggesting that EcR activation was required for PCD. To test the role of EcR-A, we examined the fate of RP2s in an $E c R-A$ null mutant $\left(E c R^{139} / E c R^{M 554 F s}\right)[74,75]$. RP2s were present in all ventral ganglia at $8 \mathrm{~h}$-APF (data not shown; $\mathrm{n}=3$ animals) and missing in neuromeres A2 to A7 when examined at $24 \mathrm{~h}-\mathrm{APF}$, while aCCs and the $\mathrm{RP} 2 \mathrm{~s}$ in A1 survived (Figure 6D; $\mathrm{n}=10$ animals). This result suggests that $\mathrm{RP} 2$ death in $\mathrm{A} 2$ to $\mathrm{A} 7$ does not require EcR-A function, and that ecdysteroids instead act via one or both EcR-B isoforms.

Having found that EcRs were present in RP2s at 8 to10 h-APF, we tested whether PCD was a direct response to prepupal ecdysteroids. Cell cultures were prepared from abdominal portions of ventral ganglia at $8 \mathrm{~h}$-APF, immediately prior to the prepupal pulse (Figure 1). In these experiments we utilized only animals with the RP2-enhanced GFP labeling pattern, in which 
the RP2s express GFP strongly and the frequency and intensity of GFP labeling in other neurons is reduced (see above). Pools of dissociated neurons were divided into paired 'sister' cultures; one culture received medium containing $6 \mu \mathrm{g} / \mathrm{ml} 20 \mathrm{E}$ while the other culture received medium without $20 \mathrm{E}$.

Immediately after flooding the dishes (designated day 0 ) we photographed fields of neurons that contained one or more putative RP2s, as identified by characteristic soma size and strong GFP labeling. We use the term 'putative RP2s' because, although cultures were prepared from animals in which GFP expression was predominantly limited to RP2s, some proportion of GFP-labeled neurons in the cultures had other identities (for example, aCCs). For each putative RP2 included in the study, we also selected a non-GFP-labeled neuron in the same field of cells, of similar size and appearance as the putative RP2, as a control neuron (not shown). The same putative RP2s and control neurons were photographed at the end of the culture period ( 48 or $72 \mathrm{~h}$ ). From photomicrographs, neurons were scored as alive or dead by morphological criteria (see Materials and methods) [24,56].

The left panels of Figure 7A, B show four putative RP2s on day 0 , with characteristic smooth, ovoid somata and well-defined nuclei. By definition, all neurons in the study were alive on day 0 . The putative RP2s cultured without $20 \mathrm{E}$ were still alive $48 \mathrm{~h}$ later (Figure 7A). As illustrated in Figure 7A, neurons that were alive after 48 or $72 \mathrm{~h}$ typically maintained strong GFP labeling. Neurons were scored as dead if the soma was rounded and shrunken or showed significant degradation, and the nucleus was no longer discernable; many shrunken neurons were also phase bright. These morphologies can be seen in the putative RP2s in Figure 7B (right panels), which were cultured in $20 \mathrm{E}$ for $48 \mathrm{~h}$. Among neurons scored as dead, GFP fluorescence was sometimes strong (Figure 7B1) and sometimes weak or absent (Figure 7B2). Accordingly, GFP fluorescence was not a useful criterion for neuronal viability and was used only to identify putative RP2s on day 0 . Note that the rounded, condensed and strongly fluorescent appearance of some neurons dying in culture (Figure 7B1, right) resembled that of RP2s dying in vivo (Figure 3A2).

Figure $7 \mathrm{C} 1$ shows the percentage of control neurons alive after 48 or $72 \mathrm{~h}$ in culture, in the presence or absence of 20E. The hormone had no significant effect on the survival of control neurons at either time point; fewer neurons were alive in older cultures but this was seen with or without $20 \mathrm{E}$. In contrast, $20 \mathrm{E}$ significantly decreased the survival of putative RP2s at both 48 and $72 \mathrm{~h}$, with approximately twice as many putative RP2s dying in cultures containing 20E (Figure 7C2). Thus, the PCD-inducing effect of $20 \mathrm{E}$ was specific to putative RP2s and not a general effect on all cultured neurons.

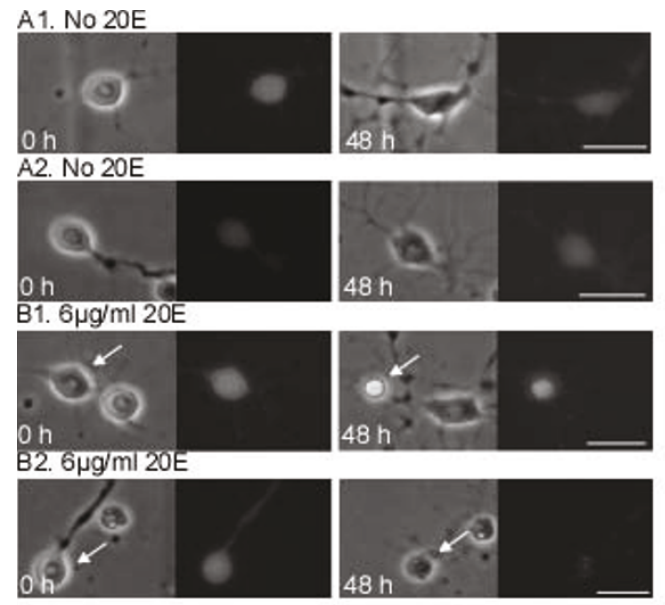

C1. Control neurons

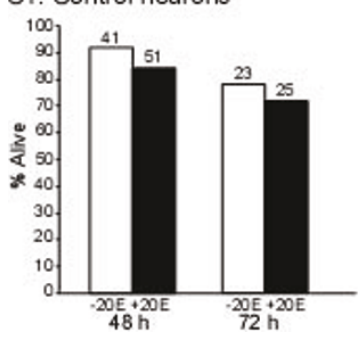

C2. Putative RP2s

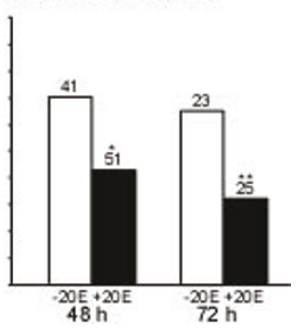

Figure 7 20E-induced programmed cell death of putative RP2s in culture. Sister cultures prepared at $8 \mathrm{~h}$-APF were treated with medium with $6 \mu \mathrm{g} / \mathrm{ml} 20 \mathrm{E}(+20 \mathrm{E})$ or no hormone (-20E). On day 0, putative RP2 neurons were identified by GFP expression and a similar-appearing neuron in the same optical field was selected to serve as a control for each putative RP2. At 48 or $72 \mathrm{~h}$ (independent experiments with four and two pairs of sister cultures, respectively) neurons were scored as dead or alive by morphological criteria. (A, B) Photomicrographs show putative RP2s at $0 \mathrm{~h}$ (left panels) and 48 h (right panels) in culture, under phase contrast (left) and GFP epifluorescence (right) optics. When multiple neurons were present, arrows mark putative RP2s. At $0 \mathrm{~h}$, all putative RP2s had smooth, ovoid somata with well-defined nuclei. (A1, A2). In the absence of 20E, putative RP2s were still alive at 48 h, with GFP labeling and neurite outgrowth (B1, B2). In the presence of $20 \mathrm{E}$, putative RP2s were dead by $48 \mathrm{~h}$, as determined by morphological criteria; the neuron in (B1) was shrunken and phase-bright but retained GFP fluorescence, while that in (B2) was shrunken, had a fragmented outline and faint GFP fluorescence, and the nucleus was reduced to a small spot. (C) Histograms show percentage of neurons alive at the time indicated; $n$ given above each bar. (C1) The percentage of control neurons alive at 48 or $72 \mathrm{~h}$ did not differ significantly between +20 and -20E cultures. (C2) At both 48 and 72 h, significantly fewer putative RP2s survived in cultures containing $20 \mathrm{E}$ $\left({ }^{*} P<0.05,{ }^{* *} P<0.01\right.$, two-tailed Chi square test with Yates continuity correction). Scale bars: $10 \mu \mathrm{m}$.

In other experiments in which putative RP2s were cultured from 'clear-gut' wandering larvae, 20E did not induce PCD of putative RP2s (data not shown). This is consistent with the developmental regulation of 20E's ability to trigger neuronal death, as also observed in Manduca [20]. 
Propidium iodide confirmation of programmed cell death To independently assess the validity of the morphological criteria for PCD, we stained neurons with propidium iodide (PI) at the end of the culture period (48 or $72 \mathrm{~h}$ ). Similarly to other vital stains such as acridine orange and trypan blue, PI is excluded from live neurons whereas membrane breakdown in dying or dead neurons allows PI to penetrate and fluorescently label nucleic acids $[66,76]$, From photomicrographs, each putative RP2 was scored independently as dead or alive by morphological criteria and PI staining. All putative RP2s were alive on day 0 (data not shown). Figure 8A, B shows representative photomicrographs of putative RP2s after $48 \mathrm{~h}$ in culture without (Figure 8A) or with (Figure 8B) 20E. The putative RP2 in Figure 8A was alive by morphological criteria and lacked PI staining ('PI-negative') while the putative RP2 in Figure 8B was dead by morphological criteria and showed PI staining ('PI-positive'). Figure $8 \mathrm{C}$ compares the results from the two scoring methods. As in the previous experiments (Figure 7C2), 20E significantly reduced the percentage of putative RP2s alive after $48 \mathrm{~h}$ (Figure $8 \mathrm{C} 1$ ) or $72 \mathrm{~h}$ (Figure $8 \mathrm{C} 2$ ) in culture. The two scoring methods - morphological criteria and PI staining - yielded nearly identical results; neurons judged to be alive by morphological criteria were PI-negative whereas neurons judged to be dead by morphological criteria were PI-positive.

Role of neuronal contacts in programmed cell death In Manduca, contact with other neurons does not influence the ability of $20 \mathrm{E}$ to trigger segment-specific PCD of cultured motoneurons [56]. The current experiments utilized relatively high-density cultures, so many putative RP2s had somatic and/or neuritic contact with other neurons. To test the hypothesis that $20 \mathrm{E}$ acts directly on RP2s to trigger PCD, independently of neuronal contact, we analyzed separately a subset of putative RP2s that lacked contact with other neurons throughout the culture period (determined by high-magnification examination of photomicrographs). Figure 9A, B shows representative live and dead contact-free RP2s at $48 \mathrm{~h}$, in the absence or presence of $20 \mathrm{E}$, respectively. In both examples the neurons' somata and neurites (fragmented in Figure 9B) failed to contact adjacent neurons. The number of putative RP2s that lacked contacts was relatively small, so we pooled data from 48- and 72-h experiments (same data as Figures 7 and 8) to identify 39 contact-free putative RP2s. Figure 9C shows that, among these contact-free putative RP2s, significantly fewer survived when cultured with 20E, consistent with the idea that neuronal contact played no role in RP2's responses to $20 \mathrm{E}$.

Taken together, the above results support the hypothesis that the rising phase of the prepupal pulse of

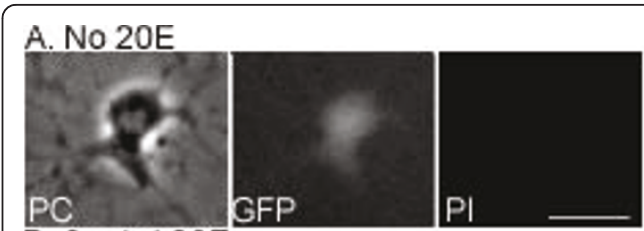

B. $6 \mu \mathrm{g} / \mathrm{ml} 20 \mathrm{E}$
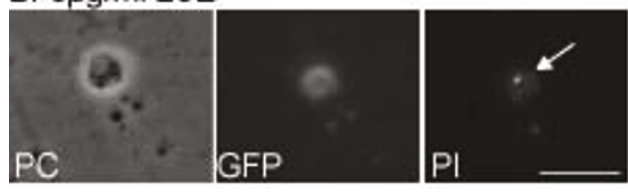

\section{C1. $48 \mathrm{~h}$ in culture}

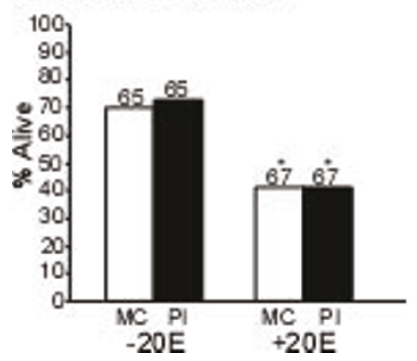

C2. $72 \mathrm{~h}$ in culture

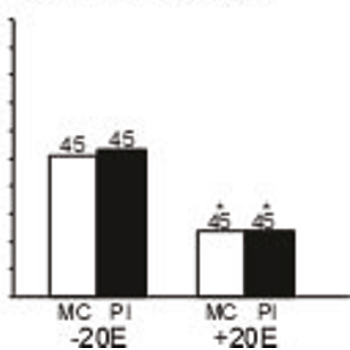

Figure 8 Validation of morphological criteria for programmed cell death by propidium iodide staining. Putative RP2s were cultured at 8 h-APF (independent experiments with six and four pairs of sister cultures, respectively) in medium with $6 \mu \mathrm{g} / \mathrm{ml} 20 \mathrm{E}$ (+20E) or no hormone (-20E). After 48 or $72 \mathrm{~h}, \mathrm{RP} 2 \mathrm{~s}$ were

independently scored as dead or alive using morphological criteria (MC) or PI staining. (A, B) Photomicrographs show putative RP2s under phase contrast (PC) and epifluorescence optics for GFP and PI (a marker for dead cells). (A) Putative RP2 cultured for $48 \mathrm{~h}$ without 20E. The neuron was alive by morphological criteria, exhibited GFP labeling and lacked PI staining. (B) Putative RP2 cultured for $48 \mathrm{~h}$ with 20E was dead by morphological criteria, exhibited GFP labeling and had strong nuclear PI staining (arrow). (C) Similar results were obtained by scoring putative RP2s by morphological criteria or PI staining after $48 \mathrm{~h}$ (C1) or $72 \mathrm{~h}$ (C2) in culture. Both methods showed that significantly fewer putative RP2s survived in cultures with $20 \mathrm{E}\left({ }^{*} P<0.01\right.$, two-tailed Chi square test with Yates continuity correction). Scale bars: $10 \mu \mathrm{m}$.

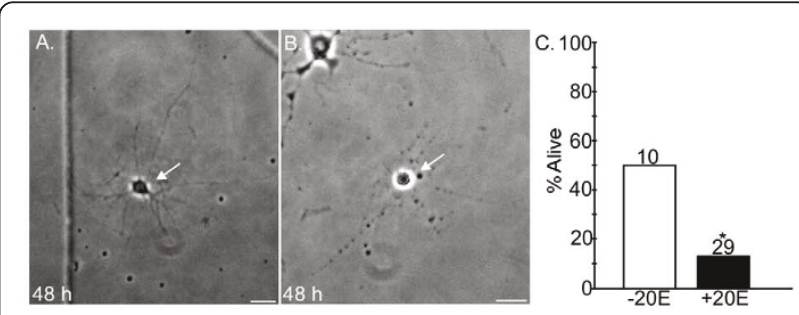

Figure 9 Cell contact does not regulate putative RP2 fate in culture. (A, B) Low-power photomicrographs show contact-free putative RP2s after $48 \mathrm{~h}$ in culture, in the absence (A) or presence (B) of 20E. The putative RP2 in (A) is alive and that in (B) is dead. Note absence of neuritic contact with other neurons. (C) Histogram shows percentage of putative RP2s alive at 48 or $72 \mathrm{~h}$ (data pooled from Figures 7 and 8); $n$ given above each bar. Among these contact-free putative RP2s, significantly fewer survived in cultures containing $20 \mathrm{E}\left({ }^{*} P=0.028\right.$; Fisher's exact test). Scale bar: $10 \mu \mathrm{m}$. 
ecdysteroids is the normal developmental trigger for PCD of RP2s in neuromeres A2 to A7 and that this effect is mediated directly.

\section{Discussion}

Previous work in Manduca used a combination of in vivo and cell culture methods to establish that the segment-specific PCD of APR motoneurons during metamorphosis is triggered directly by ecdysteroids [20]. However, the relative lack of genetic tools available in Manduca motivated us to seek a comparable experimental system in Drosophila. After investigating a variety of potential reporters, we selected the even-skipped gene combined with other constructs (see Materials and methods) to drive GFP expression in two patterns that allowed us to track neuronal fates: RP2/aCC-enhanced and RP2-enhanced (Figure 2B). The lack of restriction of GFP expression to RP2s had advantages and disadvantages: one advantage is that the survival of aCCs under all conditions provided a powerful positive control for the death of RP2s in neuromeres A2 to A7. A disadvantage is that, even when using animals with the RP2-enhanced GFP labeling pattern, cell cultures contained some GFP-labeled neurons that were not RP2s. However, despite this complication, we readily detected 20E-induced PCD in culture (Figures 7 and 8). Further refinement of the reporter lines and genetic constructs could likely remedy these current limitations. The present study establishes RP2 motoneurons as tractable and informative subjects for investigating hormonally triggered PCD during Drosophila metamorphosis, in individually identified neurons of known function.

\section{A segmental subset of RP2s is eliminated by programmed cell death}

During Drosophila metamorphosis, many larval motoneurons are remodeled or undergo PCD. We determined that the segmental subset of RP2s in neuromeres A2 to A7 dies during the larval-pupal transformation, with PCD beginning at approximately 14 to $16 \mathrm{~h}-\mathrm{APF}$, based on the disappearance of GFP-labeled RP2s in ventral ganglia (Table 1; Figure 3A). That the disappearance of GFP-labeled RP2s resulted from death rather than other causes (for example, cessation of GFP expression) was confirmed first by the presence of TUNEL labeling (indicating DNA fragmentation) in RP2s during the same time period (15 h-APF; Figure 3B) and, secondly, by the finding that genetic manipulations of known Drosophila PCD genes could block RP2's death (Figures 4 and 5; see below). Not all RP2s were TUNEL-positive at $15 \mathrm{~h}$-APF, likely due to minor temporal variability in the PCD pathway within and between animals, also seen during motoneuron death in Manduca [77]. In all experimental conditions, aCC motoneurons in A1 to
A7, and the RP2s in A1, survived this period of development (see Results).

\section{The involvement of programmed cell death genes and proteins in RP2s}

It was beyond the scope of the present study to test the potential involvement of even a major fraction of genes implicated in Drosophila PCD. We instead focused on a small subset of representative genes. In Drosophila, the death-activating genes reaper, hid, and grim collectively or independently activate PCD, in many cases by antagonizing Diap1 [49-53]. Death of RP2s may depend exclusively on Reaper, as perturbation of reaper but not hid function impaired their deaths (Figure 4). We did not test the role of Grim directly but the finding that RP2 death was impaired in reaper mutants, despite one intact copy of grim, suggests that Grim is unnecessary. This is supported by studies suggesting that PCD in the developing central nervous system is normal in grim mutants [78].

In contrast to salivary glands[62,67], we were unable to block the death of RP2s by ectopic expression of Diap1 (Figure 5A2). Likewise, in $t h^{S L}$ homozygotes in which Reaper cannot inactivate Diap1, RP2 death was normal (Figure 5A3). Thus, Reaper-mediated PCD may be Diap1-independent in RP2s as well as vCrz neurons [17]. Reaper is multi-functional, including a role in mitochondrial permeabilization[79,80], which has been shown to be required for some developmental PCD [81]. Regardless of the involvement of Diap1, caspase activation appears necessary for RP2 death in A2 to A7, as shown by the ability of the viral caspase inhibitor p35 to preserve these neurons (Figure $5 \mathrm{C}$ ). Also consistent with the role of caspases, RP2 death was blocked in animals with mutations in dark (Figure 5D), whose gene product is a key component of the apoptosome, where caspases are activated.

These results are consistent with a general requirement for $\mathrm{H} 99$ proteins, caspases and apoptosome function for embryonic and postembryonic PCD in the nervous system and other tissues of Drosophila [41,51], although cell-specific variation is observed [82]. For instance, both reaper and hid participate in death of larval salivary glands while only reaper is required for $\mathrm{PCD}$ of vCrz neurons [17,53]. Likewise, as discussed above, the role of Diap1 may vary in different situations.

\section{$20 \mathrm{E}$ activates the programmed cell death pathway in RP2s}

Several independent lines of evidence suggest that the rising phase of the prepupal pulse of ecdysteroids triggers the death of RP2s in A2 to A7: the RP2s have nuclear EcRs at this time (Figure 6A, B), genetic disruption of EcR function by overexpression of a dominant- 
negative receptor blocks RP2 death (Figure 6C) and, in cell culture, 20E treatment delivered at the time of the prepupal pulse promotes selectively the death of putative RP2s (Figures 7 and 8). The requirement for EcR function is consistent with the overall role of ecdysteroids in driving metamorphosis [7], with different isoforms often associated with different developmental events. The EcR-A isoform mediates the PCD of type II neurons at the end of metamorphosis when ecdysteroids decline [27], whereas EcR-A is not required for RP2 death (Figure 6D). One or both EcR-B isoforms must therefore be responsible, but distinguishing between them was beyond the scope of this study. In Drosophila, EcR-B isoforms are implicated in many cases of neuronal remodeling [13-15] and neuronal death, including that of vCrz neurons in response to the late larval pulse of ecdysteroids [17]. It is notable that both doomed RP2s and persisting aCCs had qualitatively similar patterns of EcR-B1 expression (Figure 6A, B), so the simple presence or absence of receptor cannot account for their different fates. Molecular players other than EcR, including EcR's heterodimerization partner, ultraspiracle (USP) [83,84], and nuclear hormone co-regulators [85], are likely important for regulating neuron- and segment-specific actions of ecdysteroids.

The prepupal pulse of ecdysteroids begins at about 10 h-APF (Figure 1) and the death of RP2s begins between 14 and 16 h-APF (Table 1; Figure 3). This delay is consistent with typical latencies for EcR-induced cellular changes mediated via gene transcription [86]. We tested the hypothesis that the rise in the prepupal pulse of ecdysteroids triggers RP2 death by preparing neuronal cultures at 8-h APF and maintaining them with or without $20 \mathrm{E}$ for 48 or $72 \mathrm{~h}$ (Figures 7 and 8). In the absence of a reporter line that restricted GFP expression to RP2s, we used animals with the RP2-enhanced GFP labeling pattern in which most but not all GFP-labeled neurons were RP2s. Furthermore, due to variation in the exact site of the cut made to separate the thoracic and abdominal regions of the ventral ganglion before dissociating neurons, some RP2s from neuromere A1 may have been included in some cultures. Both of these factors could potentially impair the ability to detect a $20 \mathrm{E}$ effect. However, the ability of 20E to induce PCD of putative APRs was robust in each of the four independent experiments shown in Figures $7 \mathrm{C} 2$ and $8 \mathrm{C} 2$; in all cases, significantly more putative RP2s died in the presence than in the absence of 20E. Furthermore, this effect was specific to these neurons as $20 \mathrm{E}$ had no significant effect on the survival of control neurons in the same culture dishes. Results were essentially identical when using morphological criteria or PI staining to score neurons as alive or dead (Figure 8). Importantly, given that $20 \mathrm{E}$ exposure was maintained for the entire culture period, the rise in ecdysteroids during the prepupal pulse appears sufficient to trigger RP2 death, without requiring a subsequent decline in ecdysteroids. The same result was obtained for cultured Manduca motoneurons [56].

Some experimental limitations should be noted. Even after a 72-h exposure to 20E, some putative RP2s failed to degenerate (Figures 7 and 8); because the percentage of dying neurons increased between 48 and 72 hours, a longer culture period may have allowed more neurons to die. Conversely, some putative RP2s died when cultured in the absence of $20 \mathrm{E}$; although all cultures were prepared at $8 \mathrm{~h}-\mathrm{APF}$, it is possible that some RP2s were already committed to PCD due to asynchrony when the prepupal pulse began. The identical phenomena were observed in Manduca, even with unambiguous identification of specific motoneurons by retrograde fluorescent labeling from their target muscle[24,54,56].

The present study identified the hormonal trigger and a few of the intermediate steps required for RP2's death during metamorphosis, but further experiments are required to determine to what extent the specific pathway for ecdysteroid-activated PCD in RP2s matches that in other Drosophila cells and tissues. Ecdysteroids and ecdysteroid-regulated early genes up-regulate Reaper and Dronc in larval midgut and salivary glands [87-91] and transcriptional co-activators or repressors that operate outside of EcR signaling (for example, Forkhead and Med24) may also be critical for the timing and execution of PCD $[92,93]$. One intriguing but unanswered question is how segmentally iterated neurons such as RP2 exhibit segment-specific responses to a generalized hormonal cue.

\section{Conclusions}

This study introduces RP2 motoneurons as a useful model for investigating how ecdysteroids regulate postembryonic PCD in Drosophila in neurons of known function. Data from in vivo and cell culture experiments suggest that the rise in ecdysteroids during the prepupal pulse acts directly, via EcR-B isoforms, to activate PCD in RP2s in abdominal neuromeres A2 to A7, while sparing RP2s in A1. Genetic manipulations suggest that RP2's death requires Reaper function, apoptosome assembly and Diap1-independent caspase activation. RP2s offer a valuable 'single cell' approach to the molecular understanding of neuronal death during insect metamorphosis and, potentially, of neurodegeneration in other contexts [94].

\section{Materials and methods Drosophila rearing}

Drosophila were reared in $25 \times 95 \mathrm{~mm}$ polystyrene vials on standard cornmeal-yeast-agar medium [95] 
with propionic acid $(4.71 \mu \mathrm{l} / \mathrm{ml}$; Sigma-Aldritch, St Louis, MO, USA) and tegosept $(7 \mu \mathrm{g} / \mathrm{ml}$; Genesee Scientific, San Diego, CA, USA), on a 12 h light/12 h dark photoperiod. The rearing temperature was $25^{\circ} \mathrm{C}$ unless noted otherwise (see below). Animals were collected at the onset of pupariation, held at $25^{\circ} \mathrm{C}$ on $\mathrm{H}_{2} \mathrm{O}$-moistened tissues in culture dishes, and staged by h-APF.

\section{Stocks and crosses}

All stocks used to maintain reporter gene expression in RP2s and aCCs were obtained from the Drosophila Stock Center (Bloomington, IN, USA). A GFP reporter was driven in RP2s and aCC (and pCCs; see Results) using flies homozygous for the RN2-Gal4, UASm CD8GFP transgenes recombinant (second or third chromosome). In these flies, Gal4, driven by upstream promoter fragments of the even-skipped gene, drove expression of membrane-bound GFP in RP2s and aCCs exclusively in embryos $[60,96]$. We crossed these to flies that were either homozygous for a UAS-FLP, Act $5 \mathrm{C}>y+>-$ Gal4 transgene recombinant (second or third chromosome) or doubly homozygous for a UASFLP (first chromosome) and Act $5 C>y+>$ Gal4, UASGFP transgenes(s) (second chromosome). In this arrangement, Gal4 fell under the control of the Actin5C promoter following the removal of an FRT cassette by UAS-driven FLP-recombinase[58,63,97]. F1 progeny from these crosses maintained postembryonic GFP expression in RP2s and aCCs and were used for experiments.

For some experiments, including those using reaper, hid, dark, diap1 and EcR mutations, animals carrying both a UAS-FLP, Act5C $>y+>$ Gal4 and RN2-Gal4, $U A S m C D 8 G F P$ recombinant chromosome within the appropriate mutant background were generated to maintain GFP expression in RP2s and aCCs. Expression was enhanced by rearing animals at $29^{\circ} \mathrm{C}$ from oviposition to the beginning of the third instar. The following deficiencies and alleles were used: $D f(3 L) H 99$ (H99) (Drosophila Stock Center), hid ${ }^{\text {P05014, }}$, thread ${ }^{\text {SL }}$, and Df (3L)XR38 (XR38) (generously supplied by Dr Kristen White, Massachusetts General Hospital), dark ${ }^{C D 4}$ (Drosophila Stock Center) [43], $E_{c} R^{139}$ and $E c R^{M 554 F s}$ (generously supplied by Dr Michael Bender, University of Georgia)[74,75,98]. The XR38/H99 transheterozygous combination produces deletion of reaper [68] while hid $^{\text {P05014 }} /$ H99 causes a loss of hid function[50,51,65]. The $E c R^{139} / E c R^{M 554 F s}$ transheterozygote results in an $E c R-A$ null. For misexpression experiments, progeny containing at least one UAS-FLP, Act5C $>y+>$ Gal4 and RN2-Gal4, UASmCD8GFP recombinant chromosome along with either UAS-p35 [70], UAS-Diap1 [99] or $U A S-E c R-B 1^{F 645 A}[73,100]$ were used.

\section{Immunolabeling and TUNEL processing}

Using fine forceps, ventral ganglia were dissected under ice-cold PBS (pH 7.2) and fixed overnight in 4\% paraformaldehyde (Electron Microscopy Sciences, Hatfield, PA, USA) in PBS at $4^{\circ} \mathrm{C}$. Immunolabeling of GFP was performed using a rabbit anti-GFP polyclonal antibody (1:500) and an Alexa Fluor 488-conjugated goat antirabbit IgG secondary antibody (1:200; Invitrogen, Eugene, OR, USA). EcR immunolabeling was performed with monoclonal antibodies 15G1a (1:10; EcR-A) or AD4.4 (1:10; EcR-B1) [5] (Developmental Studies Hybridoma Bank developed under the auspices of the NICHD and maintained by the University of Iowa, Iowa City, IA, USA). Ventral ganglia were subjected to TUNEL using the Apop Tag ${ }^{\circledR}$ Red In Situ Apoptosis Detection Kit (Chemicon Intl, Temecula, CA, USA). Ganglia were imaged on either a Bio-Rad Radiance 2100 (Bio-Rad, Hercules, CA, USA) or Zeiss Pascal LSM5 confocal microscope using a $488 \mathrm{~nm}$ excitation maximum argon laser (Alexa Fluor 488) or $543 \mathrm{~nm}$ excitation maximum helium-neon laser (rhodamine). Images were processed and converted into 8 bit bitmap images using ImageJ 1.37 (NIH, Bethesda, MD, USA).

\section{Cell culture}

Animals were collected at $8 \mathrm{~h}$-APF, immediately prior to the prepupal pulse (Figure 1). The dorsal surfaces of intact prepupae were examined under a Zeiss Axiovert 25 inverted microscope and those with strong GFP expression in RP2s, and weak or no GFP expression in aCCs (see Results), were selected for experiments. Neuronal cultures were prepared using methods modified from those described previously [12,101]. Prepupae were sterilized in $95 \%$ ethanol, rinsed in sterile $\mathrm{H}_{2} \mathrm{O}$ and dissected under a laminar flow hood in glass wells (presterilized with 95\% ethanol) containing modified Drosophila defined medium (DDM2) consisting of Ham's F12 DMEM (high glucose; Irvine Scientific, Santa Ana, CA, USA) supplemented with $1.2 \mathrm{mg} / \mathrm{ml}$ sodium bicarbonate, $20 \mathrm{mM}$ HEPES, $100 \mu \mathrm{M}$ putrescine, $30 \mathrm{nM}$ sodium selenite, $20 \mathrm{ng} / \mathrm{ml}$ progesterone, $50 \mu \mathrm{g} / \mathrm{ml}$ insulin, $100 \mu \mathrm{g} / \mathrm{ml}$ transferrin (Sigma-Aldrich) and 1\% PenStrep (Invitrogen). Ventral ganglia were removed using fine forceps after which the abdominal portion was severed from the thoracic portion using a sterile 28 gauge needle (Monoject, St Louis, MO, USA). Due to the small size of the tissue, the exact location of the cut varied within the range of the third thoracic neuromere to the second abdominal neuromere.

Either six or eight abdominal ventral ganglia were pooled, divided evenly into two groups, enzymatically treated in Rinaldini's saline [102] containing $50 \mathrm{U} / \mathrm{ml}$ of papain and $1.32 \mathrm{mM}$ L-cysteine (Sigma) for 15 minutes, rinsed three times in DDM2 and mechanically 
dissociated using a fine-tipped, fire-polished Pasteur pipette. This produced two 20- $\mu$ l suspensions, each of which was dispensed into a well formed by punching an 8-mm hole into a 35-mm culture dish and attaching with Sylgard (Dow Corning Corp., Midland, MI, USA) an alphanumeric gridded coverslip (Bellco Biotechnology, Vineland, NJ, USA) coated with Concanavalin A $(200 \mu \mathrm{g} / \mathrm{ml})($ Sigma) and laminin $(3.55 \mu \mathrm{g} / \mathrm{ml}$; Invitrogen). Each well contained $80 \mu \mathrm{l}$ DDM2, resulting in a final volume of $100 \mu$ l. Pairs of cultures prepared in this manner were termed 'sister cultures.' After 30 minutes, each dish was flooded with a 3:1 mixture of DDM2 and unconditioned neurobasal medium (Invitrogen) supplemented with B27 $(20 \mu \mathrm{l} / \mathrm{ml}$; Invitrogen) to a final volume of $3 \mathrm{ml}$. One culture of each sister pair received medium containing 20E (Sigma) at a final concentration of $6 \mu \mathrm{g} / \mathrm{ml}$ while the control culture received 20E-free medium. Cultures were maintained in a humidified $23^{\circ}$ C, $5 \% \mathrm{CO}_{2}$ ambient- $\mathrm{O}_{2}$ incubator.

Cultured neurons were photographed after flooding (day 0 ) and again at 48 or $72 \mathrm{~h}$ in vitro. Fields containing one or more putative RP2 neurons, identified by their strong GFP expression (see Results), were selected randomly and photographed under phase contrast and GFP epifluorescence optics using a Nikon Coolpix 4500 digital camera attached to a Zeiss Axiovert 25 inverted microscope. Typically, 5 to 13 fields were photographed per dish. Using the gridded coverslips, the same fields were relocated and re-photographed 48 or $72 \mathrm{~h}$ later. For PI (Invitrogen) staining, PI was added to cultures at a final concentration of $20 \mu \mathrm{g} / \mathrm{ml}$ after 48 or $72 \mathrm{~h}$ in vitro. Live cells exclude PI from their somata while the nucleic acids of dead cells label strongly. PI-treated cultures were photographed as described above and also under rhodamine epifluorescence optics.

\section{Scoring neurons as alive or dead}

Cultured neurons were scored as alive or dead by morphological criteria $[24,56]$. Live neurons had smooth somata, ovoid shapes and well-defined nuclei (see Results). Only putative RP2s that fulfilled these criteria were entered into the study on day 0 . We also examined the effect of 20E on control neurons; for each putative RP2 selected on day 0, a GFP-negative neuron of similar size and appearance to the putative RP2 and located in the same photographic field was entered into the study. By definition, all putative RP2s and control neurons were alive on day 0 . At the end of the culture period (48 or $72 \mathrm{~h}$ ), an observer blind to treatment condition was provided digital images (phase-contrast and GFP fluorescence) of each putative RP2 or control neuron on day 0 and on the final day of culture. Neurons were scored as dead if the soma was rounded and shrunken or showed significant degradation, and the nucleus was no longer discernable; many dead neurons also became uniformly phase bright (see Results). For cultures stained with PI, the observer was also provided a digital image taken under rhodamine fluorescence. These neurons were scored as dead or alive by morphological criteria and independently by the presence or absence of PI staining.

Chi square with Yates continuity correction, or Fisher exact statistical tests were used to compare the proportions of neurons surviving under different conditions.

\section{Abbreviations}

20E: 20-hydroxyecdysone; aCC: anterior corner cell; APR: accessory planta retractor; Dark: Drosophila Apaf-1 related killer; DDM2: Drosophila defined medium; Diap1: Drosophila inhibitor of apoptosis protein 1; Dronc: Drosophila Nedd2-like caspase; EcR: ecdysone receptor; GFP: green fluorescent protein; h-APF: hours after puparium formation; PBS: phosphate buffered saline; pCC: posterior corner cell; PCD: programmed cell death; PI: propidium iodide; th: thread; TUNEL: terminal deoxynucleotidyl transferase mediated dUTP nick end labeling; vCrz: ventral corazonin.

\section{Acknowledgements}

We thank Drs Kristin White and Michael Bender for generously providing fly stocks, Drs Chris Doe and Judith Eisen for critical reading of the manuscript and Dr Michelle Arbeitman for providing laboratory space for some experiments. This research was supported by an American Psychological Association Diversity Program in Neuroscience Predoctoral Fellowship and NIH DBTG 5-T32-HD007348 training support (AW), and grants from the Oregon Medical Research Foundation and Oregon Partnership for Alzheimer's Research (JCW).

\section{Author details}

${ }^{1}$ Department of Biology, Institute of Neuroscience, University of Oregon Eugene, OR, 97403-1254, USA. ${ }^{2}$ Section of Molecular and Computational Biology, Department of Biological Sciences, University of Southern California, Los Angeles, CA 90089, USA.

\section{Authors' contributions}

AW participated in the design and interpretation of the study, performed all of the experiments and drafted the manuscript. JW participated in design and interpretation of the study and manuscript writing. Both authors read and approved the final manuscript.

\section{Competing interests}

The authors declare that they have no competing interests.

Received: 1 October 2010 Accepted: 27 April 2011

Published: 27 April 2011

\section{References}

1. Tissot M, Stocker RF: Metamorphosis in Drosophila and other insects: the fate of neurons throughout the stages. Prog Neurobiol 2000, 62:89-111.

2. Truman JW, Taylor BJ, Awad TA: Formation of the adult nervous system. In The Development of Drosophila melanogaster. Edited by: Bate M, Arias AM. New York: Cold Spring Harbor Press; 1993:1245-1275.

3. Lin N, Zhang C, Pang J, Zhou L: By design or by chance: cell death during Drosophila embryogenesis. Apoptosis 2009, 14:935-942.

4. Rusconi JC, Hays R, Cagan RL: Programmed cell death and patterning in Drosophila. Cell Death Differ 2000, 7:1063-1070.

5. Talbot WS, Swyryd EA, Hogness DS: Drosophila tissues with different metamorphic responses to ecdysone express different ecdysone receptor isoforms. Cell 1993, 73:1323-1337.

6. Handler AM: Ecdysteroid titers during pupal and adult development in Drosophila melanogaster. Dev Biol 1982, 93:73-82.

7. Riddiford LM: Hormones and Drosophila development. In In The Development of Drosophila melanogaster. Edited by: Bate M, Arias AM. New York: Cold Spring Harbor Press; 1993:899-939. 
8. Brown HL, Cherbas L, Cherbas P, Truman JW: Use of time-lapse imaging and dominant negative receptors to dissect the steroid receptor control of neuronal remodeling in Drosophila. Development 2006, 133:275-285.

9. Brown HL, Truman J: Fine-tuning of secondary arbor development: the effects of the ecdysone receptor on the adult neuronal lineages of the Drosophila thoracic CNS. Development 2009, 136:3247-3256.

10. Consoulas C, Restifo LL, Levine RB: Dendritic remodeling and growth of motoneurons during metamorphosis of Drosophila melanogaster. $J$ Neurosci 2002, 22:4906-4917.

11. Consoulas C, Levine RB, Restif LL: The steroid hormone-regulated gene Broad Complex is required for dendritic growth of motoneurons during metamorphosis of Drosophila. J Comp Neurol 2005, 485:321-337.

12. Kraft $R$, Levine $R B$, Restifo $L$ : The steroid hormone 20-hydroxyecdysone enhances neurite growth of Drosophila mushroom body neurons isolated during metamorphosis. J Neurosci 1998, 18:8886-8899.

13. Lee T, Marticke S, Sung C, Robinow S, Luo S: Cell-autonomous requirement of the USB/ECR-B ecdysone receptor for mushroom body neuronal remodeling in Drosophila. Neuron 2000, 28:807-818.

14. Schubiger M, Wade AA, Carney GE, Truman JW, Bender M: Drosophila EcR$B$ ecdysone receptor isoforms are required for larval molting and for neuron remodeling during metamorphosis. Development 1998, 125:2053-2062.

15. Schubiger $M$, Tomita S, Sung C, Robinow S, Truman JW: Isoform specific control of gene activity in vivo by the Drosophila ecdysone receptor. Mech Dev 2003, 120:909-918.

16. Zheng X, Wang J, Haerry TE, Wu AY, Martin J, O'Connor MB, Lee CH, Lee T: TGF-beta signaling activates steroid hormone receptor expression during neuronal remodeling in the Drosophila brain. Cell 2003, 112:303-315.

17. Choi YJ, Lee G, Park JH: Programmed cell death mechanisms of identifiable peptidergic neurons in Drosophila melanogaster. Development 2006, 133:2223-2232.

18. Levine RB, Truman JW: Dendritic reorganization of abdominal motoneurons during metamorphosis of the moth, Manduca sexta>. $J$ Neurosci 1985, 5:2424-2431

19. Prugh J, Della Croc K, Levine RB: Effects of the steroid hormone 20hydroxyecdysone, on the growth of neurites by identified insect motoneurons in vitro. Dev Biol 1992, 154:331-347.

20. Weeks JC: Thinking globally, acting locally: steroid hormone regulation of the dendritic architecture, synaptic connectivity and death of an individual neuron. Prog Neurobiol 2003, 70:421-442.

21. Weeks JC, Ernst-Utzschneider K: Respecification of larval proleg motoneurons during metamorphosis of the tobacco hornworm, Manduca sexta: segmental dependence and hormonal regulation. $J$ Neurobiol 1989, 20:569-592

22. Weeks JC, Truman JW: Independent steroid control of the fates of motoneurons and their muscles during insect metamorphosis. J Neurosci 1985, 5:2290-2300

23. Weeks JC, Roberts W, Trimble DL: Hormonal regulation of segmental specificity of motoneuron phenotype during metamorphosis of the tobacco hornworm, Manduca sexta. Dev Biol 1992, 149:185-196.

24. Zee MC, Weeks JC: Developmental change in the steroid hormone signal for cell-autonomous, segment-specific programmed cell death of a motoneuron. Dev Biol 2001, 235:45-61.

25. Kimura K, Truman JW: Postmetamorphic cell death in the nervous and muscular systems of Drosophila melanogaster. J Neurosci 1990, 10:403-411.

26. Draizen TA, Ewer J, Robinow S: Genetic and hormonal regulation of the death of peptidergic neurons in the Drosophila central nervous system. $J$ Neurobiol 1999, 38:455-465.

27. Robinow S, Talbot WS, Hogness DS, Truman JW: Programmed cell death in the Drosophila CNS is ecdysone-regulated and coupled with a specific ecdysone receptor isoform. Development 1993, 119:1251-1259.

28. Robinow S, Draizen TA, Truman JW: Genes that induce apoptosis: transcriptional regulation in identified, doomed neurons of the Drosophila CNS. Dev Biol 1997, 190:206-213.

29. Choi MK, Fahrbach SE: Demonstration of motoneuron-12 sparing in cultured Manduca sexta ventral nerve cords. J Neurobiol 1992, 23:364-375.

30. Choi MK, Fahrbach SE: Evidence for an endogenous neurocidin in the Manduca sexta ventral nerve cord. Arch Insect Biochem Physiol 1995, 28:273-289.
31. Hay BA, Guo M: Caspase-dependent cell death in Drosophila. Annu Rev Cell Dev Biol 2006, 22:623-650

32. Chew SK, Akdemir F, Chen P, Lu W-J, Mills K, Daish T, Kumar S, Rodriguez A, Abrams JM: The apical caspase dronc governs programmed and unprogrammed cell death in Drosophila. Dev Cell 2004, 7:897-907.

33. Daish TJ, Mills K, Kumar S: Drosophila caspase DRONC is required for specific developmental cell death pathways and stress-induced apoptosis. Dev Cell 2004, 7:909-915.

34. Dorstyn L, Colussi PA, Quinn LM, Richardson H, Kumar S: DRONC, an ecdysone-inducible Drosophila caspase. Proc Natl Acad Sci USA 1999, 96:4307-4312.

35. Waldhuber M, Emoto K, Petritsch C: The Drosophila caspase DRONC is required for metamorphosis and cell death in response to irradiation and developmental signals. Mech Dev 2005, 122:914-927.

36. Xu D, Li Y, Arcara M, Lackey M, Bergmann A: The CARD-carrying caspase Dronc is essential for most, but not all, developmental cell death in Drosophila. Development 2005, 132:2125-2134.

37. Muro I, Hay BA, Clem RJ: The Drosphila DIAP1 protein is required to prevent accumulation of a continuously generated, processed form of the apical caspase DRONC. J Biol Chem 2002, 277:49644-49650.

38. Quinn LM, Dorstyn L, Mills K, Colussi PA, Chen P, Coombe M, Abrams J, Kumar S, Richardson H: An essential role for the caspase Dronc in developmentally programmed cell death in Drosophila. J Biol Chem 2000, 275:40416-40424.

39. Yan N, Huh JR, Schirf V, Demeler B, Hay BA, Shi Y: Structure and activation mechanism of the Drosophila initiator caspase Dronc. J Biol Chem 2006, 281:8667-8674.

40. Yu X, Wang L, Acehan D, Wang X, Akey CW: Three-dimensional structure of a double apoptosome formed by the Drosophila Apaf-1 related killer. J Mol Biol 2006, 355:577-589.

41. Akdemir F, Farkaš R, Chen P, Juhasz G, Medved'ová L, Sass M, Wang L, Wang X, Chittaranjan S, Gorski SM, Rodriguez A, Abrams JM: Autophagy occurs upstream or parallel to the apoptosome during histolytic cell death. Development 2006, 133:1457-1465.

42. Mills K, Daish T, Harvey KF, Pfleger CM, Hariharan IK, Kumar S: The Drosophila melanogaster Apaf-1 homologue ARK is required for most, but not all, programmed cell death. J Cell Biol 2006, 172:809-815.

43. Rodriguez A, Oliver H, Zou H, Chen P, Wang X, Abrams JM: Dark is a Drosophila homologue of Apaf-1/CED-4 and functions in an evolutionarily conserved death pathway. Nat Cell Biol 1999, 1:272-279.

44. Bergmann A: The role of ubiquitylation for the control of cell death in Drosophila. Cell Death Differ 2010, 17:61.

45. Bergmann A, Yang AY, Srivastava M: Regulators of IAP function: coming to grips with the grim reaper. Curr Opin Cell Biol 2003, 15:717-724.

46. Meier P, Silke J, Leevers SJ, Evan Gl: The Drosophila caspase DRONC is regulated by DIAP1. EMBO J 2000, 19:598-611.

47. Muro I, Hay BA, Clem RJ: The Drosphila DIAP1 protein is required to prevent accumulation of a continuously generated, processed form of the apical caspase DRONC. J Biol Chem 2002, 277:49644-49650.

48. Wilson R, Goyal L, Ditzel M, Zachariou A, Baker DA, Agapite J, Steller $\mathrm{H}$, Meier P: The DIAP1 RING finger mediates ubiquitination of Dronc and is indispensable for regulating apoptosis. Nat Cell Biol 2002, 4:445-450.

49. Chen P, Nordstrom W, Gish B, Abrams JM: grim, a novel cell death gene in Drosophila. Genes Dev 1996, 10:1773-1782.

50. Grether ME, Abrams JM, Agapite J, White K, Steller H: The head involution defective gene of Drosophila melanogaster functions in programmed cell death. Genes Dev 1995, 9:1694-1708.

51. White K, Grether ME, Abrams JM, Young K, Farrell K, Steller H: Genetic control of programmed cell death in Drosophila. Science 1994, 264:677-683.

52. Goyal L McCall K, Agapite J, Hartwieg E, Steller H: Induction of apoptosis by Drosophila reaper, hid and grim through inhibition of IAP function. EMBO J 2000, 19:589-597.

53. Yin VP, Thummel CS: A balance between the diap1 death inhibitor and hid death inducers controls steroid-triggered cell death in Drosophila. Proc Natl Acad Sci USA 2004, 101:8022-8027.

54. Hoffman KL, Weeks JC: Programmed cell death of an identified motoneuron in vitro: temporal requirements for steroid exposure and protein synthesis. J Neurobiol 1998, 35:300-322. 
55. Hoffman KL, Weeks JC: Role of caspases and mitochondria in the steroidinduced programmed cell death of a motoneuron during metamophosis. Dev Biol 2001, 229:517-536.

56. Streichert LC, Pierce JT, Nelson JA, Weeks JC: Steroid hormones act directly to trigger segment-specific programmed cell death of identified motoneurons in vitro. Dev Biol 1997, 183:95-107.

57. Choi JC, Park D, Griffith LC: Electrophysiological and morphological characterization of identified motor neurons in the Drosophila third instar larva central nervous system. J Neurophysiol 2004, 91:2353-2365.

58. Hartwig CL, Worrell J, Levine RB, Ramaswam M, Sanyal S: Normal dendrite growth in Drosophila motor neurons requires the AP-1 transcription factor. Dev Neurobiol 2008, 68:1225-1242.

59. Sink H, Whitington PM: Location and connectivity of abdominal motoneurons in the embryo and larva of Drosophila melanogaster. $J$ Neurobiol 1991, 22:298-311.

60. Fujioka M, Lear BC, Landgraf M, Yusibova GL, Zhou J, Riley KM: Evenskipped, acting as a repressor, regulates axonal projections i Drosophila. Development 2003, 130:5385-5400

61. Goodman CS, Bastiani MJ, Doe CQ, du Lac S, Helfand SL, Kuwada JY, Thomas JB: Cell recognition during neuronal development. Science 1984, 225:1271-1279.

62. Winbush A: Steroid-triggered, cell-autonomous programmed cell death of identified Drosophila motoneurons during metamorphosis. PhD thesis University of Oregon, Biology Department; 2008.

63. Duffy JB, Harrison DA, Perrimon N: Identifying loci required for follicular patterning using directed mosaics. Development 1998, 125:2263-2271.

64. Gavrieli Y, Sherman Y, Ben-Sasson SA: Identification of programmed cell death in situ via specific labeling of nuclear DNA fragmentation. J Cell Biol 1992, 119:493-501.

65. Peterson C, Carney GE, Taylor BJ, White K: reaper is required for neuroblast apoptosis during Drosophila development. Development 2002, 129:1467-1476.

66. Jiang C, Baehrecke EH, Thummel CS: Steroid regulated programmed cell death during Drosophila metamorphosis. Development 1997, 124:4673-4683.

67. Yin VP, Thummel CS, Bashirullah A: Down-regulation of inhibitor of apoptosis levels provides competence for steroid-triggered cell death. J Cell Biol 2007, 178:85-92.

68. Lisi S, Mazzon I, White K: Diverse domains of THREAD/DIAP1 are required to inhibit apoptosis induced by REAPER and HID in Drosophila. Genetics 2000, 154:669-678

69. Bump NJ, Hackett M, Hugunin M, Seshagiri S, Brady K, Chen P, Ferenz C, Franklin S, Ghayur T, Li P, Licari P, Mankovich J, Shi L, Greenberg AH, Miller LK, Wong WW: Inhibition of ICE family proteases by baculovirus antiapoptotic protein p35. Science 1995, 269:1885-1888.

70. Hay BA, Wolff T, Rubin GM: Expression of baculovirus P35 prevents cell death in Drosophila. Development 1994, 120:2121-2129.

71. Mills K, Daish T, Kumar S: The function of the Drosophila caspase DRONC in cell death and development. Cell Cycle 2005, 4:744-746.

72. Truman JW, Talbot WS, Fahrbach SE, Hogness DS: Ecdysone receptor expression in the CNS correlates with stage-specific responses to ecdysteroids during Drosophila and Manduca development. Development 1994, 120:219-234.

73. Hu X, Cherbas L, Cherbas P: Transcriptional activation by the ecdysone receptor (ECR/USP): identification of activation functions. Mol Endocrinol 2003, 17:716-731.

74. Carney GE, Robertson A, Davis MB, Bender M: Creation of EcR isoformspecific mutations in Drosophila melanogaster via local $\mathrm{P}$ element transposition, imprecise $\mathrm{P}$ element excision, and male recombination. Mol Gen Genomics 2004, 271:282-290.

75. Davis MB, Carney GE, Robertson AE, Bender M: Phenotypic analysis of EcR$A$ mutants suggests that $\mathrm{EcR}$ isoforms have unique functions during Drosophila development. Dev Biol 2005, 282:385-396.

76. Kamikubo Y, Takaori-Kondo A, Uchiyama T, Hori T: Inhibition of cell growth by conditional expression of kpm, a human homologue of Drosophila warts/lats tumor. J Biol Chem 2003, 278:17609-176014.

77. Kinch G, Hoffman KL, Rodrigues EM, Zee MC, Weeks JC: Steroid-triggered programmed cell death of a motoneuron is autophagic and involves structural changes in mitochondria. J Comp Neurol 2003, 457:384-403.

78. Wu JN, Nguyen N, Aghazarian M, Tan Y, Sevrioukov EA, Mabuchi M, Tang W, Monserrate JP, White K, Brachmann CB: grim promotes programmed cell death of Drosophila microchaete glial cells. Mech Dev 2010, 127:407-417.

79. Thomenius M, Kornbluth S: Multifunctional reaper: sixty-five amino acids of fury. Cell Death Differ 2006, 13:1305-1309.

80. Abdelwahid E, Yokokura T, Krieser RJ, Balasundaram S, Fowle WH, White K: Mitochondrial disruption in Drosophila apoptosis. Dev Cell 2007, 12:793-806.

81. Goyal G, Fell B, Sarin A, Youle RJ, Sriram V: Role of mitochondrial remodeling in programmed cell death in Drosophila melanogaster. Dev Cell 2007, 12:807-816.

82. Bangs $\mathrm{P}$, White K: Regulation and execution of apoptosis during Drosophila development. Dev Dyn 2000, 218:68-79.

83. Hall BL, Thummel CS: The RXR homolog ultraspiracle is an essential component of the Drosophila ecdysone receptor. Development 1998, 125:4709-4717.

84. Yao TP, Segraves WA, Oro AE, McKeown M, Evans RM: Drosophila ultraspiracle modulates ecdysone receptor function via heterodimer formation. Cell 1992, 71:63-72.

85. Wolf IM, Heitzer MD, Grubisha M, DeFranco DB: Coactivators and nuclear receptor transactivation. J Cell Biochem 2008, 104:1580-1586.

86. Thummel CS: Molecular mechanisms of developmental timing in C. elegans and Drosophila. Dev Cell 2001, 1:453-465.

87. Cakouros D, Daish T, Martin D, Baehrecke EH, Kumar S: Ecdysone-induced expression of the caspase DRONC during hormone-dependent programmed cell death in Drosophila is regulated by Broad-Complex. $J$ Cell Biol 2002, 157:985-995

88. Cakouros D, Daish T, Kumar S: Ecdysone receptor directly binds the promoter of the Drosophila caspase dronc, regulating its expression in specific tissues. J Cell Biol 2004, 165:631-640.

89. Jiang C, Lambin A-FJ, Steller H, Thummel CS: A steroid-triggered transcriptional hierarchy controls salivary gland cell death during Drosophila metamorphosis. Mol Cell 2000, 5:445-455.

90. Lee C-Y, Wendel DP, Reid P, Lam G, Thummel CS: E93 directs steroidtriggered programmed cell death in Drosophila. Mol Cell 2000, 6:433-443.

91. Lee C-Y, Cooksey BAK, Baehrecke EH: Steroid regulation of midgut cell death during Drosophila development. Dev Biol 2002, 250:101-111.

92. Cao C, Liu Y, Lehmann M: Fork head controls the timing and tissue selectivity of steroid-induced developmental cell death. J Cell Biol 2007, 176:843-852.

93. Wang L, Lam G, Thummel CS: Med24 and Mdh2 are required for Drosophila larval salivary gland cell death. Dev Dyn 2010, 239:954-964.

94. Lu B, Vogel H: Drosophila models of neurodegenerative diseases. Annu Rev Pathol 2009, 4:315-342.

95. Lewis EB: A new standard food medium. Dros Info Serv 1960, 34:117-118.

96. Lee T, Luo L: Mosaic analysis with a repressible cell marker for studies of gene function in neuronal morphogenesis. Neuron 1999, 22:451-461.

97. Ito K, Awano W, Suzuki K, Hiromi Y, Yamamoto D: The Drosophila mushroom body is a quadruple structure of clonal units each of which contains a virtually identical set of neurons and glial cells. Development 1997, 124:761-771

98. Bender M, Imam FB, Talbot WS, Ganetzky B, Hogness DS: Drosophila ecdysone receptor mutations reveal functional differences among receptor isoforms. Cell 1997, 91:777-788.

99. Hay BA, Wassarman DA, Rubin GM: Drosophila homologs of baculovirus inhibitor of apoptosis proteins function to block cell death. Cell 1995, 83:1253-1262.

100. Cherbas L, Hu X, Zhimulev I, Belyaeva E, Cherbas P: EcR isoforms in Drosophila: testing tissue-specific requirements by targeted blockade and rescue. Development 2003, 130:271-284.

101. Su H, O'Dowd DK: Fast synaptic currents in Drosophila mushroom body Kenyon cells are mediated by a-bungarotoxin-sensitive nicotinic acetylcholine receptors and picrotoxin-sensitive GABA receptors. J Neurosci 2003, 23:9246-9253.

102. Wu CF, Suzuki N, Poo MM: Dissociated neurons from normal and mutant Drosophila larval central nervous system in cell culture. J Neurosci 1983, 3:1888-1899.

doi:10.1186/1749-8104-6-15

Cite this article as: Winbush and Weeks: Steroid-triggered, cellautonomous death of a Drosophila motoneuron during metamorphosis. Neural Development 2011 6:15. 